\title{
Intelligent Agent Technologies: The Work Horse of ERP E-Commerce
}

\author{
Anne T. Galante \\ Department of Computing Systems, SUNY Farmingdale State College, Farmingdale, USA \\ Email: galanta@farmingdale.edu
}

Received 9 June 2015; accepted 28 July 2015; published 31 July 2015

Copyright (C) 2015 by author and Scientific Research Publishing Inc.

This work is licensed under the Creative Commons Attribution International License (CC BY). http://creativecommons.org/licenses/by/4.0/

(c) (i) Open Access

\begin{abstract}
Agents are the new defacto standard for inclusion in modules of today's software systems such as ERP systems, mobile applications and operating systems. Agents are an integral part of today's software design. The question is how do intelligent agents work in the specific area of ERP credit card processing e-commerce models? To answer this question, a specific area of ERP systems will be analyzed: credit card processing for merchants. One specific merchant credit card processor will be specifically investigated: EVO Merchants. This paper will research how exactly does ERP systems interact using Application Programing Interface or "API" specified by a credit card clearing house. Secure Socket Layers or SSL, and XML are discussed and elaborated on specifically how intelligent agents play such a pivotal role in ERP e-commerce systems for credit card processing.
\end{abstract}

\section{Keywords}

Intelligent Agents Agent Technologies, ERP, E-Commerce, API, Artificial Intelligence, Decision Making, Enterprise Resource Planning, Application Programing Interface, EVO, Credit Cards, XML, SSL, AIM, W3C, SGML

\section{Introduction}

Agent technologies are the foundation of software applications that are used in everyday life. Agents are quite flexible and because of this specific attribute, it gives them a particular strength. While agents have a particular quiet and unobtrusive characteristic, they play a crucial hand running in the background or performing automotive activities for specific users. Agents are essential to the current Enterprise Resource Planning or ERP systems specifically in today's environment where the use of credit cards payments is so prevalent.

According to the 2013 Federal Reserve Payment Study, the number of noncash payments (credit, and debit 
cards excluding electronic funds transfers or "EFT") in 2012 reached a whopping 122.8 billion of transactions, with a value of $\$ 79.0$ trillion dollars [1]. For ERP systems, agent technologies play a pivotal role in the processing of credit and debit cards.

MIT research first introduced the concept of agent technology in 1961. Agent technologies are found in a vast scope of applications ranging in the field of information sciences, computer science, specifically artificial intelligence, and other diverse traditional areas of science. According to Jennings and Wooldridge [2], an agent is located in a particularly complex environment that is an encapsulated system. An agent's primary focus is its main design objective and because of this, is goal driven and often at times collaborative.

Agents are the new defacto standard for inclusion in modules of today's software systems such as ERP systems, mobile applications and operating systems. Agents are an integral part of today's software design. The question is what advantages to agents give to a software engineer? To answer this question a specific area of ERP systems will be analyzed: credit card processing for merchants.

Agents are helpful because they can perform a variety of tasks, and thus are very flexible. According to Rusbridge [3], agents are responsible for the functions of observation, recognition, planning and or inference and action or execution. Agents are customized for application in complex systems, such as ERP systems. Some of the tasks agents accomplish are the ability to translate, communicate and publish information. Agents also can guide the user's search query from the interface to the appropriate target. Agents also negotiate, and gain access to exchange information with other agents. This negotiation is similar to a conversation that allows agents to determine which tasks are performed within the context of pre-determined tasks. These specialized features allow for the effective management of the user's environment. Agents are systems that facilitate different areas in ERP systems, such as Supply Chain or "SC" and Customer Relationship Management or "CRM" are can be viewed as a network of autonomous and collaborative units that regulate, control and organize all activities contained in the ERP system [4].

Prior research on intelligent agent system architectures has shown that problems exist within highly distributed systems that require synergy of many elements; a solution is multi-agent systems or "MAS" [5] [6]. Additional research investigated intelligent agents and ERP scheduling systems for taxi companies. The conclusion was that agents are a palpable solution for a large taxi company's complex scheduling system in London. This solution gave the ability to schedule a taxi and provide the form of payment by credit card, but it did not go into detail how the agents were involved in the credit card processing in the ERP systems [7]. Exactly how the agent works with credit card processing merchants, the ERP software is the focus of this research.

The intelligent agent plays a pivotal role in the function of using a credit card in an ERP system to pay for goods. The example of a customer ordering goods from an e-commerce site from a specific merchant, where the ERP order application would then call an Application Programing Interface or "API" specified by a credit card clearing house. The API will process the customer's credit or debit card information. An example of a company who provides these types of specifications for API for credit cards is EVO Payments International. EVO founded in 1989, and EVO's corporate office is located in New York. EVO Payments International is among the largest fully integrated merchant acquirer and payment processors in the world. EVO operates as a payment service provider for both face-to-face and e-commerce transactions for all major credit cards, debit cards, commercial cards and electronic bank transfers. EVO can process in nearly 50 markets and 120 currencies around the world. Through its European subsidiary, EVO operates as a principal member of MasterCard Worldwide and Visa Europe [8].

\section{Problem Definition}

Some of the specific tasks of the EVO credit card system, related to intelligent agents, will be subsequently addressed and discussed. How does agent technology interface with ERP systems and credit card processing merchants? These questions are discussed in the research.

\section{Advanced Integration Method-AIM}

The API tool EVO provides is a merchant web services API, Advanced Integration Method or "AIM". AIM provides the necessary protocols to connect an e-commerce site or a retail point of sale to the Authorize Net Payment Gateway to submit credit card information by activating the AIM API. AIM will validate credit card information, provide a receipt of the transaction, and will secure all credit card information using a 128 bit Secure Socket Layer or "SSL". According to Bhiogade [9] SSL is a protocol developed by Netscape that estab- 
lishes a secure communication for a web browser and a web server. The SSL protocol requires the web server to have a digital certificate installed in order for the SSL connection to be created. SSL works by using a public key to encrypt the data transferred over the SSL. Figure 1 shows the process on how to implement AIM.

Figure 1 begins with Step 1 with a developer creating an application or modifying an existing application to obtain the necessary credit card information. In Step 2, a secure SSL connection is established to EVO's payment gateway. The developer must parse the credit card information into a format specified by AIM. The results are passed back to the merchant software application to initially initiated the transaction.

The parsed credit card information is embedded in an Extensible Markup Language or "XML" document. According to Shanmugasundaram, Tufte, Zhang, He, DeWitt, \& Naughton [10], XML is a class of data objects called XML documents and partially and subsequently describes the behavior of the computer programs that are responsible for processing the documents. XML is profile of Standard Generalized Markup Language or "SGML" defined by the World Wide Web Consortium or W3C. The World Wide Web Consortium or "W3C" is an international community run by member organizations, a full-time staff, and the public. The members work together to develop Web standards. Led by Web inventor Tim Berners-Lee and CEO Jeffrey Jaffe, W3C's mission is to lead the Web to its full potential [11]. XML documents contained parsed data and are made up of character data called entities. Markup is the encoded description of the documents storage layout and logical structure. Figure 2 is an example of test data parsed and encoded in XML.

Figure 2 shows the parsed data collected from the program developed by the software engineer for the purpose of credit card processing. Each piece of data is put into a markup, or encoded, with beginning and ending tags. This is similar to the Hyper Text Markup Language or "HTML" works. For example, the beginning structure is $<$ MerchantAuthentication $>$. The merchant name is parsed with $<$ name $>$ and an ending $<$ /name $>$ tag. The actual merchant name is placed in the markup.
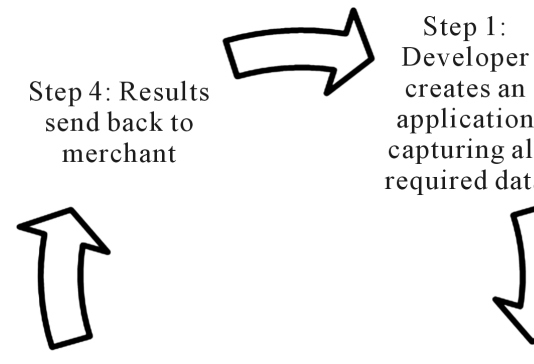
required data

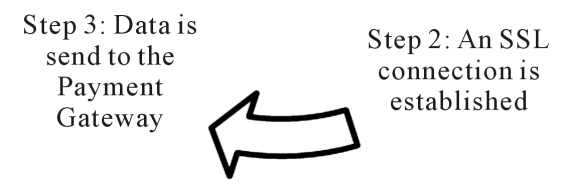

Figure 1. Example of the payment gateway process.

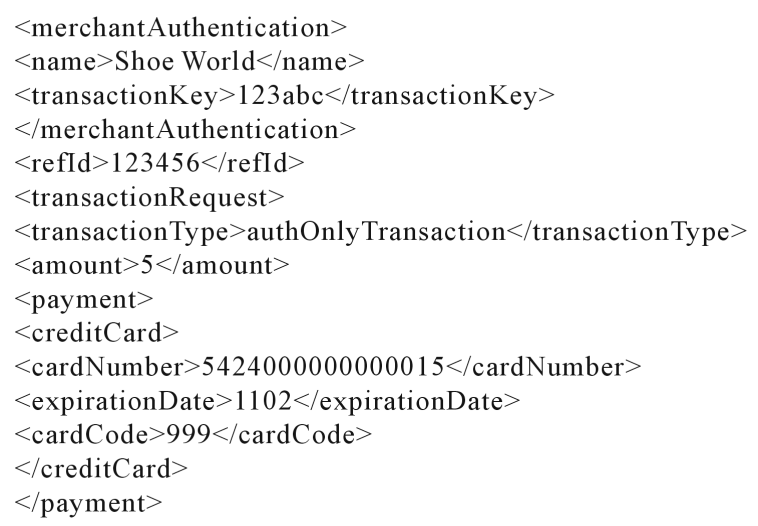

Figure 2. Example sample test XML data. 


\section{Conclusions}

AIM is an API provided by EVO for merchants to install in ERP systems. There are four steps to credit card approval (parse data, secure SSL, send data, receive a response). Intelligent agents are the workers of the corporate ERP systems, transferring data and performing services established in the software framework. In order to implement AIM, a software developer must write a program or modify and already established system to obtain the necessary credit card information. A SSL certificate is installed on the server. The data is parsed with XML according the vendor's specifications. The data is transmitted to the payment gateway and the AIM API goes to work. AIM validates the data, processes it, and sends results back to the receiver.

The paper researched how the implementation of the AIM API for merchant credit card processing is seamless, transparent, and unobtrusive to users. The use of agent technology gives maximum control over the merchants credit card processing. The implementation of agents in complex environments is a palpable solution for merchants with e-commerce sites. This solution is innovative, scalable, and compatible with today's modern trends for optimization and effectiveness.

\section{References}

[1] (2013) Federal Reserve Bank Service. https://www.frbservices.org/files/communications/pdf/research/2013_payments_study_summary.pdf

[2] Wooldridge, M. and Jennings, N.R. (1995) Intelligent Agents: Theory and Practice. The Knowledge Engineering Review, 10, 115-152. http://dx.doi.org/10.1017/S0269888900008122

[3] Rusbridge, C. (1998) Towards the Hybrid Library. https://www.era.lib.ed.ac.uk/bitstream/handle/1842/1736/DLib98.pdf;jsessionid=54AF601B4AC4262188CE469427A A8FC3? sequence $=2$

[4] Symeonidis, A.L., Kehagias, D.D. and Mitkas, P.A. (2003) Intelligent Policy Recommendations on Enterprise Resource Planning by the Use of Agent Technology and Data Mining Techniques. Expert Systems with Applications, 25, 589-560. http://dx.doi.org/10.1016/S0957-4174(03)00099-X

[5] Jennings, N.R., Sycara, K. and Wooldridge, M.J. (1998) A Roadmap of Agent Research and Development. Autonomous Agents and Multi-Agent Systems. Kluwer Academic, Boston.

[6] Ferber, J. (1999) Multi-Agent Systems: An Introduction to Distributed Artificial Intelligence. Addison Wesley Longman, Harlow.

[7] Glaschenko, A., Ivaschenko, A., Rzevski, G. and Skobelev, P. (2009) Multi-Agent Real Time Scheduling System for Taxi Companies. Proceedings of the 8th International Conference on Autonomous Agents and Multiagent Systems, Budapest, 10-15 May 2009, 29-36.

[8] (2015) EVO Payments International. http://evopayments.com/about-evo/evo-payments-international

[9] Bhiogade, M.S. (2001) Secure Socket Layer. Proceedings of the Computer Science and Information Technology Education Conference, Cork, June 2002, 85-90. http://dx.doi.org/10.2139/ssrn.291499

[10] Shanmugasundaram, J., Tufte, K., Zhang, C., He, G., DeWitt, D.J. and Naughton, J.F. (1999) Relational Databases for for Querying XML Documents: Limitations and Opportunities. Proceedings of the 25th International Conference on Very Large Data, 1999, 302-314.

[11] (2015) About W3C. http://www.w3.org/Consortium/ 